\title{
Inherited bone marrow failure syndromes
}

\author{
Inderjeet Dokal
}

Received: 21 April 2011 / Accepted: 28 April 2011 /Published online: 13 May 2011

(C) Springer-Verlag 2011

\begin{abstract}
The inherited bone marrow failure syndromes are a diverse group of disorders characterized by BM failure usually in association with one or more somatic/physical abnormality. Over the last two decades, the genes responsible for many of these have been identified. The significant advances in their molecular basis have provided insights into several biological pathways, such as DNA repair and telomere maintenance, of importance in human physiology. They have also provided a link between the inherited and idiopathic forms of bone marrow failure syndromes; for example, some patients with idiopathic forms of aplastic anemia have mutations in genes first identified in the classic forms of dyskeratosis congenita. Clinical similarities (bone marrow failure, developmental anomalies, and cancer) between these syndromes have been observed for several years; it is no surprise that some overlap is also observed at the level of molecular pathology. For example, Shwachman-Diamond syndrome and Diamond-Blackfan anemia both appear to be disorders of ribosome biogenesis, and patients with Fanconi anemia and dyskeratosis congenita are characterized by having short telomeres. It is possible that further overlap/connections in these pathways will emerge. The genetic advances have already led to improved diagnosis particularly where presentation is atypical. These advances may also lead to the development of new treatments.
\end{abstract}

I. Dokal $(\bowtie)$

Centre for Paediatrics, Blizard Institute of Cell and Molecular

Science, Barts and The London School of Medicine and Dentistry,

Queen Mary University of London,

Blizard Building 4 Newark Street,

Whitechapel, London E1 2AT, UK

e-mail: i.dokal@qmul.ac.uk
Keywords Congenital amegakaryocytic thrombocytopenia $\cdot$ Diamond-Blackfan anemia $\cdot$ DNA repair $\cdot$ Dyskeratosis congenita $\cdot$ Fanconi anemia $\cdot$ Inherited bone marrow failure - Ribosome biogenesis .

Shwachman-Diamond syndrome $\cdot$ Telomerase $\cdot$ Telomeres

\section{Introduction}

The inherited bone marrow (BM) failure syndromes constitute a diverse group of disorders characterized by BM failure usually in association with one or more somatic/ physical abnormality $[1,2]$. The BM failure (which can be single or multi-lineage) frequently presents in childhood, but this may be in adulthood in some cases. Furthermore, some patients initially labeled as "idiopathic aplastic anemia" are cryptic presentations of these genetic syndromes. Over the last two decades, there have been significant advances in the genetics of these syndromes with 40 genes identified to date. These advances have provided a better understanding of normal hematopoiesis and how this is disrupted in patients with BM failure. They have also provided important insights into fundamental biological pathways: DNA repair-FA/BRCA pathway, telomere maintenance-dyskeratosis congenita-related genes, ribosome biogenesis Shwachman-Diamond syndrome (SDS), and Diamond-Blackfan anemia (DBA) genes. Furthermore, as these disorders are usually associated with developmental abnormalities and an increased risk of malignancy, they are providing insights into normal human development and the genesis of cancer.

The true incidence and natural history of these disorders remains largely unknown. It is generally regarded that severe congenital neutropenia (SCN) and DBA are amongst the most prevalent of these disorders; the estimated annual 
birth incidence of DBA is 5 per $1,000,000$. The features of some of the characterized inherited BM syndromes are summarized in Tables 1 and 2. An outline of each syndrome is given below:

\section{Fanconi anemia}

Fanconi anemia (FA) is usually inherited as an autosomal recessive (AR) trait, but in a rare subset, it can be an Xlinked recessive (XLR) disorder. FA patients are clinically very heterogeneous. Characteristic features include the progressive development of BM failure and an increased predisposition to malignancy [3]. Affected individuals may also have one or more somatic abnormality including skin, skeletal, genitourinary, gastrointestinal, and neurological abnormalities. Approximately, a third of FA patients have no overt somatic abnormalities. The majority of patients present towards the end of the first decade of life. However, increasingly, some patients are being first diagnosed in adulthood, and many patients diagnosed in childhood are surviving into adulthood.

The cumulative incidence of BM failure by the age of 40 years is estimated to be around $90 \%$. At birth, the blood count is usually normal. Pancytopenia develops insidiously and presents in most cases between the ages of 5 and 10 years. In some cases, the pancytopenia develops in adolescence or even in adult life. The hemoglobin and platelet count are usually first to fall; the granulocytes are usually well-preserved in the early stages. As the pancytopenia develops, the BM becomes progressively hypocellular. There is often a marked increase in macrophage activity with evidence of hemophagocytosis. BM failure leading to fatal hemorrhage or infection is the main cause of death in FA patients.
FA is associated with an increased risk of myelodysplasia, leukemia, and non-hematological malignancies. The leukemias are usually of the acute myeloid type, particularly FAB types M4 (myelomonocytic) and M5 (monocytic). In some cases, leukemia may be the initial event leading to the diagnosis of FA. The cumulative incidence of hematological malignancy by the age of 40 years is $33 \%$. In addition to the hematological malignancies, there is a significant risk of hepatic tumors and squamous cell carcinoma, including squamous cell carcinomas of the vulva, esophagus, head, and neck. The cumulative incidence of solid tumors is calculated to be $28 \%$ by the age of 40 years.

FA cells display a high frequency of spontaneous chromosomal breakage and hypersensitivity to DNA cross-linking agents such as diepoxybutane (DEB) and mitomycin-c (MMC). This genomic instability led to the development of a diagnostic test (the "DEB/MMC stress test") over two decades ago and has facilitated many advances, including elucidation of the genetics with 14 subtypes/complementation groups currently characterized. The proteins encoded by the FA genes (Table 2) participate in a complicated network important in DNA repair [4-6] (Fig. 1). Specifically, eight of the FA proteins (FANCA, FANCB, FANCC, FANCE, FANCF, FANCG, FANCL, and FANCM) interact with each other and form a nuclear complex called the "FA core complex". The FA core complex is required for the activation of the FANCIFANCD2 protein complex to a monoubiquitinated form (FANCI-FANCD2-Ub). FANCI-FANCD2-Ub then interacts with DNA repair proteins (including BRCA2, BRCA1, and RAD51) leading to repair of the DNA damage. FA-D1 patients have biallelic mutations in BRCA2. These observations have linked the FA proteins with BRCA1 and BRCA2 (FANCD1) in a DNA damage response pathway,

Table 1 Characteristics of the inherited bone marrow failure syndromes

\begin{tabular}{|c|c|c|c|c|c|c|}
\hline & FA & $\mathrm{DC}$ & SDS & DBA & CAMT & $\mathrm{SCN}$ \\
\hline Inheritance pattern & AR, XLR & XLR, AR AD & $\mathrm{AR}$ & $\mathrm{AD}$ & $\mathrm{AR}$ & $\mathrm{AD}, \mathrm{AR}$ \\
\hline Somatic abnormalities & Yes & Yes & Yes & Yes & Rare & Rare \\
\hline Bone marrow failure & AA $(>90 \%)$ & AA $(\sim 80 \%)$ & AA $(\sim 20 \%)$ & $\mathrm{RCA}^{\mathrm{a}}$ & $\mathrm{Meg}^{\mathrm{b}}$ & Neut $^{\mathrm{c}}$ \\
\hline Short telomeres & Yes & Yes & Yes & No & $?$ & $?$ \\
\hline Cancer & Yes & Yes & Yes & Yes & Yes & Yes \\
\hline Chromosome instability & Yes & Yes & Yes & $?$ & $?$ & $?$ \\
\hline Genes identified & 14 & 8 & 1 & 9 & 1 & 4 \\
\hline
\end{tabular}

FA Fanconi anemia, DC dyskeratosis congenita, SDS Shwachman-Diamond syndrome, DBA Diamond-Blackfan anemia, $C A M T$ congenital amegakaryocytic thrombocytopenia, $S C N$ severe congenital neutropenia, $A D$ autosomal dominant, $A R$ autosomal recessive, $X L R$ x-linked recessive, $R C A$ red cell aplasia, $M e g$ megakaryocytes, Neut neutrophils

${ }^{\text {a }}$ Red cell aplasia although some patients can develop global BM failure

${ }^{\mathrm{b}}$ Low megakaryocytes which can progress to global BM failure

${ }^{\mathrm{c}}$ Usually low neutrophils 
Table 2 Inherited BM failure genetic subtypes

(a) FA complementation groups/genetic subtypes

Complementation group/gene

A $(F A N C A)$

$\mathrm{B}\left(F A N C B^{\mathrm{a}}\right)$

$\mathrm{C}(F A N C C)$

$\mathrm{D} 1\left(F A N C D 1^{\mathrm{b}}\right)$

D2 (FANCD2)

$\mathrm{E}($ FANCE)

$\mathrm{F}(F A N C F)$

$\mathrm{G}(F A N C G)$

I $(F A N C I)$

$\mathrm{J}\left(F A N C J / B R I P 1^{\mathrm{c}}\right)$

$\mathrm{L}($ FANCL)

$\mathrm{M}(F A N C M)$

$\mathrm{N}\left(\right.$ FANCN/PALB2 $\left.2^{\mathrm{d}}\right)$

$\mathrm{P}($ FANCP/SLX4)

(b) DC genetic subtypes

DC Subtype

$\mathrm{X}$-linked recessive

Autosomal dominant

Autosomal dominant

Autosomal recessive

Uncharacterized $^{\mathrm{e}}$

(c) SDS genetic subtypes

SDS Subtype

Autosomal recessive

Uncharacterized

(d) DBA genetic subtypes

DBA Subtype

Autosomal dominant

Uncharacterized $^{\mathrm{f}}$

(e) CAMT genetic subtypes

CAMT Subtype

Autosomal recessive

Uncharacterized

(f) SCN genetic subtypes

Subtype

Autosomal dominant
Approximate $\%$ of FA patients

65

$<1$

12

$<1$

$<1$

4

4

12

$<1$

$<5$

$<1$

$<1$

$<1$

$?$

Approximate $\%$ of DC patients

30

5

5

10

$<1$

$<1$

$<1$

1

2

40

Approximate $\%$ of SDS patients

$>90$

$<10$

Approximate $\%$ of DBA patients

25

2

1

7

5

3

1

7

3

$40-50$

Approximate $\%$ of CAMT patients

$?$

$?$

Approximate $\%$ of patients

$50-60$

$<1 \%$
Chromosome location

$16 \mathrm{q} 24.3$

Xp22.2

9q22.3

$13 q 12.3$

$3 \mathrm{p} 25.3$

$6 \mathrm{p} 21.3$

$11 \mathrm{p} 15$

$9 \mathrm{p} 13$

$15 \mathrm{q} 26.1$

$17 \mathrm{q} 23.1$

$2 \mathrm{p} 16.1$

$14 q 21.3$

$16 \mathrm{p} 12.1$

$16 \mathrm{p} 13.3$

Chromosome location

$\mathrm{Xq} 28$

$3 \mathrm{q} 26$

$5 \mathrm{p} 15.33$

$14 \mathrm{q} 12$

$15 \mathrm{q} 14$

$5 \mathrm{p} 15.33$

$5 \mathrm{q} 35.3$

$17 \mathrm{p} 13.1$

16q21

?

Chromosome location

7q11.21

?

Chromosome location

$19 \mathrm{q} 13.2$

10q22

$15 \mathrm{q} 25.2$

$1 \mathrm{p} 22.1$

1p35-36.1

$3 \mathrm{q} 29$

$2 \mathrm{p} 25$

$6 \mathrm{p} 21.31$

$12 \mathrm{q} 13$

?

Chromosome location $1 \mathrm{p} 34$

?

Chromosome location

19 p 13.3

$1 \mathrm{p} 22$

$\begin{array}{ll}\text { Gene product } & \text { Exons } \\ \text { FANCA } & 43 \\ \text { FANCB } & 10 \\ \text { FANCC } & 14 \\ \text { FANCD1 } & 27 \\ \text { FANCD2 } & 44 \\ \text { FANCE } & 10 \\ \text { FANCF } & 1 \\ \text { FANCG } & 14 \\ \text { FANCI } & 35 \\ \text { FANCJ } & 20 \\ \text { FANCL } & 14 \\ \text { FANCM } & 23 \\ \text { FANCN } & 13 \\ \text { FANCP } & 15\end{array}$

Gene product Exons

Dyskerin 15

TERC 1

TERT 16

TIN2 6

NOP10 2

TERT 16

NHP2 4

TCAB1 10

C16orf57 7

?

Gene product Exons

SBDS 5

?

Gene product Exons

RPS19 6

RPS24 7

RPS17 5

RPL5 8

RPL11 6

RPL35A 5

RPS7

RPS10

RPS26

?

Gene product Exons

MPL

?

?

Gene product Exons

ELA2 5

GFI1 7 
Table 2 (continued)

\begin{tabular}{lllll}
\hline Autosomal recessive & $10-15$ & $1 \mathrm{q} 21.3$ & HAX1 & 7 \\
& $?$ & $17 \mathrm{q} 21.31$ & G6PC3 & $?$ \\
Uncharacterized $^{\mathrm{g}}$ & 30 & $?$ & $?$ \\
\hline
\end{tabular}

${ }^{\mathrm{a}} F A N C B$ is on the $\mathrm{X}$-chromosome

${ }^{\mathrm{b}}$ FANCD1 is BRCA2

${ }^{\mathrm{c}}$ FANCJ is BRIP1 (partner of BRCA1)

${ }^{\mathrm{d}}$ FANCN is PALB2 (partner of BRCA2)

${ }^{\mathrm{e}}$ These are likely to represent more than one genetic locus and include the genetically heterogeneous AR DC

${ }^{\mathrm{f}}$ These are likely to represent more than one genetic locus

${ }^{\mathrm{g}}$ This is likely to be a heterogeneous group. Furthermore, some patients initially presenting as isolated neutropenia may turn out to be cryptic presentation of other syndromes such as Wiskot-Aldrich syndrome and Shwachman-Diamond syndrome

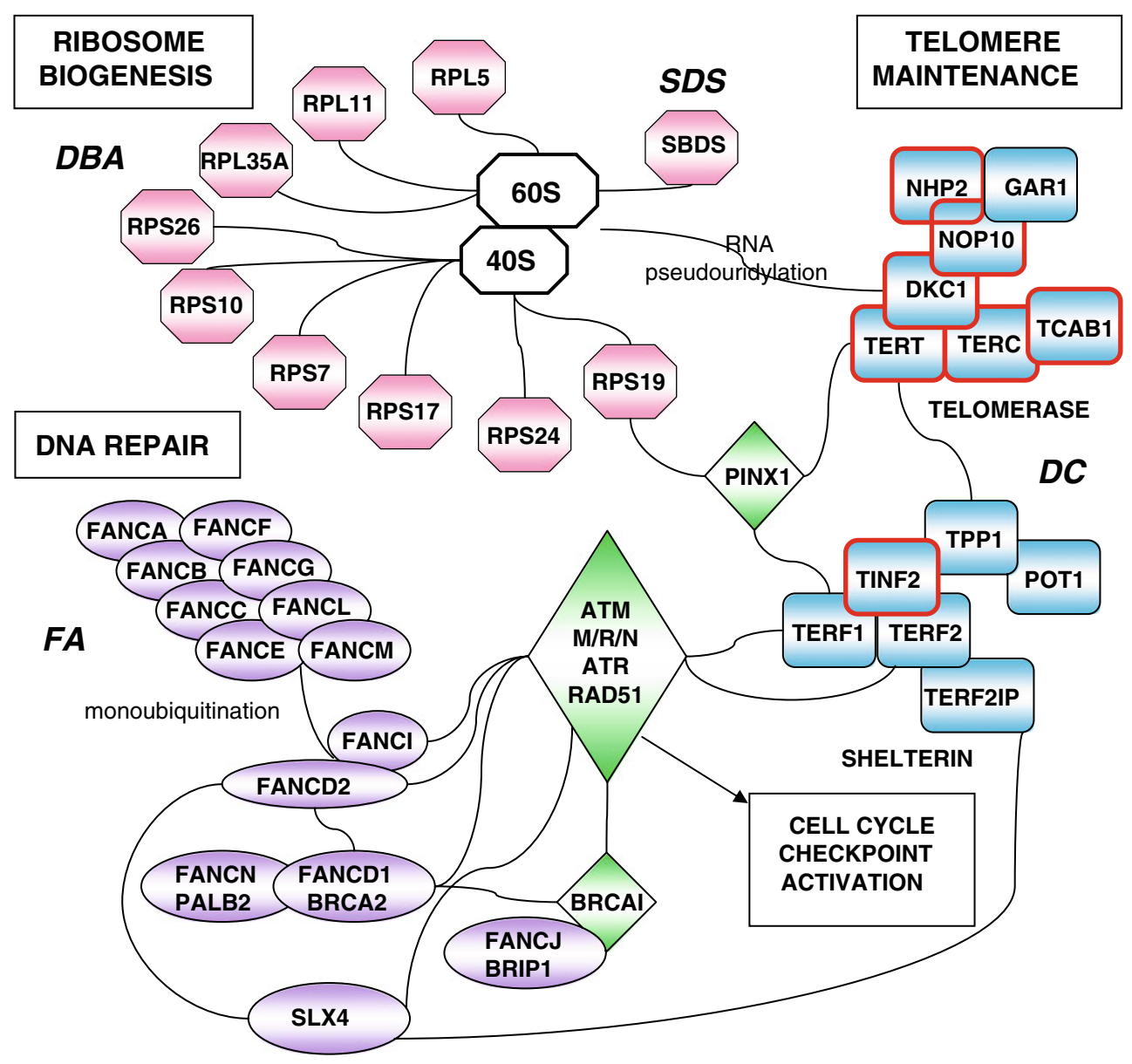

Fig. 1 Interconnected pathways associated with bone marrow failure. Genes known to be mutated in three different pathways that lead to bone marrow failure are highlighted. Genes mutated in the telomere maintenance pathway are circled in red. Interactions are as defined in Entrez Gene at http://www.ncbi.nlm.nih.gov/. Gene names for those mutated as well as for several key interconnecting proteins are as follows: RP ribosomal protein; FANC, Fanconi anemia complementation group; DKC1, dyskeratosis congenita 1, dyskerin; NOP10, nucleolar protein 10 homolog; NHP2, non-histone ribonucleoprotein 2 homolog; GAR1, glycine and arginine rich ribonucleoprotein 1 homolog; TCAB1, telomerase cajal body protein 1; TERF1, telomeric repeat binding factor 1; TERF2, telomeric repeat binding factor 2;
TINF2, TERF1-interacting nuclear factor 2; TERF2IP, TERF2 interacting protein (RAP1); POT1 protection of telomeres 1 homolog; TPP1, TIN2 interacting protein $1(=\mathrm{ACD}$, adrenocortical dysplasia homolog); PINX1, PIN2 (=TERF1) interacting protein; ATM, ataxia telangiectasia mutated; $\mathrm{M} / \mathrm{R} / \mathrm{N}=\mathrm{MRE} 11 / \mathrm{RAD} 50 / \mathrm{NBS} 1$, meiotic recombination 11 homolog $\mathrm{A} /$ radiation resistance 50 homolog/ Nijmegen breakage syndrome 1; ATR, ataxia telangiectasia and Rad3 related (Seckel syndrome); BRCA1, breast cancer 1. BRIP1, BRCA1 interacting protein C-terminal helicase 1; PALB2, partner and localizer of BRCA2; SLX4, structure-specific endonuclease subunit. Figure modified from reference 2 and 37 
"The FA/BRCA pathway". The BRCA2 protein is important in the repair of DNA damage by homologous recombination. Cells lacking BRCA2 inaccurately repair damaged DNA and are hypersensitive to DNA crosslinking agents. It has also been observed that FANCJ is BRIP1 (partner of BRCA1) and that FANCN is PALB2 (partner of BRCA2). These findings further strengthen the connection between FA and DNA repair. Very recently, it has been established that SLX4 (an endonuclease which is an important regulator of DNA repair) is also an FA protein [7]; biallelic mutations in SLX4 were observed in FA-P patients.

\section{Dyskeratosis congenita}

Classic dyskeratosis congenita (DC) is an inherited BM failure syndrome characterized by the muco-cutaneous triad of abnormal skin pigmentation, nail dystrophy, and mucosal leucoplakia [8, 9]. A variety of other (dental, gastrointestinal, genitourinary, neurological, ophthalmic, pulmonary, and skeletal) abnormalities have also been reported. BM failure is the major cause of premature mortality $(\sim 70-80 \%)$ with an additional predisposition to malignancy $(\sim 10 \%)$ and fatal pulmonary complications ( 10-15\%). XLR, autosomal dominant (AD), and AR subtypes of DC are recognized.

$\mathrm{BM}$ failure occurs at a high frequency in $\mathrm{DC}$; by the age of 30 years, approximately $80 \%$ of patients will have developed some degree of BM failure. As in other forms of $\mathrm{BM}$ failure, there is a variable reduction in the marrow cellularity and hematopoietic stem/progenitors. Myelodysplasia and leukemia are also observed in DC patients. Non-hematological malignancies (e.g., carcinomas of the upper gastro-intestinal tract) occur at a much higher frequency compared to the normal population. Like FA, DC patients, therefore, also have a high predisposition to developing malignancy.

DC cells grow poorly in vitro, but they usually do not exhibit hypersensitivity to DNA cross-linking agents. Chromosomal abnormalities (particularly rearrangements) can be seen in DC cells, and therefore, like FA, it can be regarded as a chromosome instability disorder. Eight DC genes (DKC1, TERC, TERT, NOP10, NHP2, TINF2, $T C A B 1$, and C16orf57) have been identified to date [1013]. Seven of these are important in telomere maintenance, and DC is now considered to be principally a disorder of telomere maintenance.

The gene mutated in X-linked DC $(D K C 1)$ encodes a highly conserved nucleolar protein called dyskerin. Dyskerin associates with the H/ACA class of small nucleolar RNAs in small nucleolar ribonucleoprotein particles (snoRNPs), which are important in guiding the conversion of uracil to pseudouracil during the maturation of ribosomal RNA [14]. Dyskerin also associates with the RNA component of telomerase (TERC), where it is important in stabilizing the telomerase complex, which is critical in the maintenance of telomeres [15]. Heterozygous mutations in TERC and TERT (telomerase reverse transcriptase) have been found in patients with AD-DC and in some patients with aplastic anemia (AA), myelodysplasia (MDS), pulmonary fibrosis, and acute myeloid leukemia. A subset of patients with the multi-system disorder Hoyeraal-Hreidarsson ( $\mathrm{HH})$ syndrome, have been found to have $D K C 1$ mutations. It has also been established that AR-DC is genetically heterogeneous with five subtypes due to biallelic mutations in NHP2, NOP10, TERT, C16orf57, and TCAB1. One AD-DC subtype was found to be due to heterozygous mutations in TINF2 which encodes a component (TIN2) of the shelterin complex that protects telomeres and controls access to the telomere [16]. Collectively, these findings have demonstrated that classical DC, HH, a subset of AA, and MDS/AML are principally due to a defect in telomere maintenance, and cells from these patients have very short telomeres. The multi-system abnormalities seen in these patients (the "telomereopathies"), including the increased incidence of malignancy, have highlighted the critical role of telomeres and telomerase (Fig. 1) in humans.

\section{Shwachman-Diamond syndrome}

Shwachman-Diamond syndrome (SDS) is an AR disorder characterized by exocrine pancreatic insufficiency, BM failure, and other somatic abnormalities (particularly metaphyseal dysostosis) [17]. Features of pancreatic insufficiency are apparent early in infancy and frequently associated with failure to thrive. The spectrum of hematological abnormalities observed in SDS patients includes neutropenia $(>80 \%)$, anemia ( $80 \%)$, pancytopenia ( 20\%), MDS, and leukemia $(\sim 20 \%)$. The hematological complications may sometimes first develop in adulthood; the oldest reported case of AML in a patient with SDS was at age 43 years. This highlights the importance of adult hematologists to be aware of these inherited syndromes. Curiously, the incidence of leukemia seems to be higher in males compared to females (M:F; 3:1), and there is a high occurrence of isochromosome $7 \mathrm{q}$ abnormalities. Features of dysplasia and complex karyotypic changes are common in SDS associated leukemia.

The majority $(>90 \%)$ of SDS patients have been found to have biallelic mutations in the $S B D S$ gene [18]. The $S B D S$ gene product (SBDS) has an important role in the maturation of the $60 \mathrm{~S}$ ribosomal subunit and therefore ribosome biogenesis [19] (Fig. 1). 


\section{Diamond-Blackfan anemia}

Diamond-Blackfan anemia (DBA) usually presents in early infancy, with features of anemia. The hallmark of classical DBA is a selective decrease in erythroid precursors in the $\mathrm{BM}$ and normochromic macrocytic anemia associated with a variable number of somatic abnormalities such as craniofacial, thumb, cardiac, and urogenital malformations [20]. In many patients $(>50 \%)$, there will be no obvious physical anomalies, and this can pose diagnostic difficulties. MDS and AML have been reported in a few patients, suggesting an increased predisposition to hematological malignancies. There are also cases that have evolved into AA. Thus, although DBA has been regarded classically as a pure red cell aplasia, a more global hematopoietic defect can be observed, particularly in older patients.

The first DBA gene (RPS19) was identified in 1999 [21] and in Western populations accounts for $\sim 25 \%$ of DBA patients. Subsequently, heterozygous mutations (consistent with autosomal dominant inheritance) in other genes encoding ribosomal proteins of the small (RPS24, RPS17, RPS7, RPS10, RPS26) and large (RPL5, RPL11, RPL35A) ribosomal subunits have also been reported; collectively, the genetic basis of $\sim 50-60 \%$ of DBA patients can now be established [21, 22]. These observations have also demonstrated that DBA is a disorder of ribosome biogenesis (Fig. 1). It is noteworthy that patients with mutations in the RPL5 gene tend to have multiple physical abnormalities, including craniofacial, thumb, and heart anomalies, whereas isolated thumb malformations are predominantly seen in patients with heterozygous RPL11 mutations.

In the Japanese, RPS19 mutations only account for $\sim 13 \%$ of DBA patients, and there are also some differences in the clinical phenotypes associated with the different DBA genes compared to western populations [23]. This suggests ethnic differences in phenotypic expression; a feature that has been observed previously in other genetic diseases including FA. It also highlights the need to define the clinical-genetic spectrum in different ethnic populations.

It is noteworthy that DBA has been demonstrated to be caused by mutations in genes important in the biogenesis of the small and large ribosomal units. Secondly, that the SBDS gene mutated in SDS (see above) is required for the maturation of the large ribosomal unit. Furthermore, the 5q-syndrome (a recognized subtype of acquired myelodysplasia) is associated with haploinsufficiency of the gene encoding the ribosomal protein RPS14 [24]. These advances have thus provided a very interesting connection between DBA, SDS, MDS, and defective ribosome biogenesis. They also highlight the importance of studies on rare syndromes as often as they can provide significant insights into the pathology of more common diseases.

\section{Severe congenital neutropenia (including Kostmann syndrome)}

Severe congenital neutropenia (SCN) is heterogeneous and is characterized by peripheral neutropenia $\left(<0.2 \times 10^{9} / 1\right)$ [25]. These patients characteristically present with recurrent life-threatening infections in infancy. Usually, they have no overt somatic/physical anomalies. BM examination typically reveals a maturation arrest in the myeloid lineage. These patients can progress to myelodysplasia and leukemia, usually with acquisition of secondary mutations including the granulocyte colony stimulating factor (G-CSF) receptor. In the majority of patients, heterozygous mutations in neutrophil elastase gene (ELA2) have been demonstrated [26]. These mutations are thought to lead to accumulation of the nonfunctional protein, which in turn triggers an unfolded protein response leading to maturational arrest. The original family described by Kostmann, had AR SCN, and has been shown to be associated with biallelic mutations in $H A X 1$ gene predicted to lead to defects in cell death [27]. Mutations in other genes (GFI1, G6PC3,WASP) are also known to be associated with SCN demonstrating genetic heterogeneity [28].

\section{Congenital amegakaryocytic thrombocytopenia}

Congenital amegakaryocytic thrombocytopenia (CAMT) usually presents in infancy and is characterized by isolated thrombocytopenia, reduction/absence of megakaryocytes in the BM with usually no somatic abnormalities [29]. Approximately $50 \%$ of patients will develop AA usually by the age of 5 years. They can also evolve into MDS or leukemia. In a subgroup of CAMT patients, biallelic mutations in the gene encoding for the thrombopoietin receptor $(M P L)$ have been identified [30].

\section{Treatment of the hematopoietic complications in the inherited BM failure syndromes}

Major advances in supportive care have led to considerable improvement in the outcome of these patients. Blood product support has been a key component of this effort. Red cell transfusions should be given to maintain the hemoglobin at a level which allows the patient to be asymptomatic (typically $>10 \mathrm{~g} / \mathrm{dl}$ ), platelets should be maintained above $10 \times 10^{9} / 1$. It is reasonable to give prophylactic antimicrobials to patients with a neutrophil count below $0.5 \times 109 / 1$ and who are receiving immunosuppressive therapy. As a general rule, all patients need prompt therapy with broad-spectrum intravenous antibiotics if they develop an infection. Leuco-depleted and where appropriate CMV-negative, blood products should be chosen to prevent 
development of HLA antibodies and reduce the risk of CMV disease.

Inherited bone marrow failure syndromes do respond to specific interventions. In patients with DBA, the first line therapy remains corticosteroids (usually prednisolone). Up to $80 \%$ of patients respond to steroids as a single agent. The dose and frequency of prednisolone is titrated to the lowest amount required to maintain a hemoglobin response to minimize side effects. In patients who are refractory to steroids, or when patients lose steroid responsiveness, supportive treatment with regular blood transfusions is instituted. This should be accompanied with a comprehensive iron chelating program to prevent iron overload. At this stage, for DBA patients who have a compatible sibling bone marrow donor, hematopoietic stem cell transplantation (SCT) may become appropriate and is potentially curative.

The mainstay of management of neutropenia in SCN patients is with G-CSF. More than $>90 \%$ respond to treatment, and the dose is adjusted to maintain an absolute neutrophil count of more than $1.5-2.0 \times 10^{9} / 1$. For patients failing G-CSF therapy or progressing to leukemia, SCT may be appropriate and curative.

In patients with FA and DC and who have significant peripheral cytopenia $\left(\mathrm{Hb}<8 \mathrm{~g} / \mathrm{dl}\right.$, neutrophils $<0.5 \times 10^{9} / 1$, platelets $<20 \times 10^{9} / 1$ ), the first line medical therapy is usually with oxymetholone. This can be started at a dose of $0.5-1.0 \mathrm{mg} / \mathrm{kg} / \mathrm{day}$ and gradually increased, if necessary, to a maximal dose of $5 \mathrm{mg} / \mathrm{kg}$. In general, DC patients can respond to lower doses of oxymetholone compared to FA patients. Patients with severe disease and a HLA-compatible donor should go onto receive a SCT at the earliest opportunity if they become refractory to oxymetholone.

It has been recognized that patients with inherited BM failure syndromes merit special considerations when undergoing hematopoietic SCT. Some of these patients (especially FA) are hypersensitive to agents such as cyclophosphamide and irradiation compared to non-FA patients [31, 32]. This is not surprising given that the genetic defect associated with FA is in DNA repair mechanisms. Low toxicity conditioning regimens especially using fludarabine-based protocols and low dose or avoiding radiotherapy provide better survival rates in these patients. This represents a major advance in outcome following SCT in FA patients [33, 34]. Lowintensity fludarabine-based SCT protocols are also producing better results in patients with $\mathrm{DC}[35,36]$.

Acknowledgments I would like to thank my current (Richard Beswick, Upal Hossain, Michael Kirwan, Amanda Walne, and Tom Vulliamy) and past colleagues (Stuart Knight, Anna Marrone, Philip Mason, and David Stevens) whose contribution has been important to our research program over the years. I am also grateful to the patients and all our colleagues (doctors and nurses) for their support and to the Wellcome Trust for the financial support.

\section{References}

1. Alter BP, Young NS (1998) The bone marrow failure syndromes. In: Nathan DG, Orkin HS (eds) Hematology of infancy and childhood. WB Saunders, Philadelphia, pp 237-335

2. Dokal I, Vulliamy $T$ (2010) Inherited bone marrow failure syndromes. Haematologica 95(8):1236-1240

3. Auerbach AD, Buchwald M, Joenje H (2001) In: CR Scriver et al. (eds)The metabolic and molecular basis of inherited disease, McGraw-Hill, New York pp753-768

4. Joenje H, Patel KJ (2001) The emerging genetic and molecular basis of Fanconi anaemia. Nat Rev Genet 2:446-457

5. Wang W (2007) Emergence of a DNA-damage response network consisting of Fanconi anaemia and BRCA proteins. Nat Rev Genet 6:735-748

6. Kee Y, D'Andrea AD (2010) Expanding roles of the Fanconi anemia pathway in preserving genomic stability. Genes Dev 24:1680-1694

7. Alderton GK (2011) Expanding the reach of Fanconi anaemia. Nat Rev Canc 11:158

8. Dokal I (2000) Dyskeratosis congenita in all its forms. Br J Haematol 110:768-779

9. Bessler M, Wilson DB, Mason PJ (2010) Dyskeratosis congenita. FEBS Lett 584:3831-3838

10. Walne A, Dokal I (2009) Advances in the understanding of dyskeratosis congenita. Br J Haematol 145:164-172

11. Young NS (2010) Telomere biology and telomere diseases: implications for practice and research. Hematol Am Soc Hematol Edu Program 2010:30-35

12. Walne AJ, Vulliamy T, Beswick R, Kirwan M, Dokal I (2010) Mutations in C16orf57 and normal length telomeres unify a subset of patients with dyskeratosis congenita, poikiloderma with neutropenia and Rothmund-Thomson syndrome. Hum Mol Genet 19:4453-4461

13. Zhong F, Savage SA, Shkreli M et al (2011) Disruption of telomerase trafficking by TCAB1 mutation causes dyskeratosis congenita. Genes Dev 25:11-16

14. Meier UT (2006) How a single protein complex accommodates many different H/ACA RNAs. Trends Biochem Sci 31:311315

15. Blasco MA (2007) Telomere length, stem cells and aging. Nat Chem Biol 3:640-649

16. de Lange $\mathrm{T}$ (2005) Shelterin: the protein complex that shapes and safeguards human telomeres. Genes Dev 19:2100-2110

17. Dror Y, Freedman MH (2002) Shwachman-Diamond syndrome. Br J Haematol 118:701-713

18. Boocock GRB, Morrison JA, Popovic M et al (2003) Mutations in SBDS are associated with Shwachman-Diamond syndrome. Nat Genet 33:97-101

19. Menne TF, Goyenechea B, Sanchez-Puig N et al (2007) The Shwachman-Bodian Diamond syndrome protein mediates translational activation of ribosomes in yeast. Nat Genet 39:486496

20. Vlachos A, Ball S, Dahl N et al (2008) Diagnosis and treating Diamond Blackfan anaemia: results of an international clinical consensus conference. Br J Haematol 142:859-876

21. Draptchinskaia N, Gustavsson P, Andersson B et al (1999) The gene encoding ribosomal protein S19 is mutated in DiamondBlackfan anaemia. Nat Genet 21:169-175

22. Doherty L, Sheen MR, Vlachos A et al (2010) Ribosomal protein genes RPS10 and RPS26 are commonly mutated in DiamondBlackfan anemia. Am J Hum Genet 86:222-228

23. Konno Y, Toki T, Tandai S et al (2010) Mutations in the ribosomal protein genes in Japanese patients with Diamond-Blackfan anemia. Haematologica 95(8):1293-1299 
24. Ebert BL, Pretz J, Bosco J et al (2008) Identification of RPS14 as a $5 \mathrm{q}-$ syndrome gene by RNA interference screen. Nature 451 (7176):335-339

25. Welte K, Zeidler C (2009) Severe congenital neutropenia. Hematol Oncol Clin North Am 23:307-320

26. Dale DC, Person RE, Bolyard A et al (2000) Mutations in the gene encoding neutrophil elastase in congenital and cyclic neutropenia. Blood 96:2317-2322

27. Klein C, Grudzien M, Appaswamy G et al (2006) HAX1 deficiency causes autosomal recessive severe congenital neutropenia (Kostmann disease). Nat Genet 39:86-92

28. Person RE, Li FQ, Duan Z (2003) Mutations in proto-oncogene GFI1 cause human neutropenia and target ELA2. Nat Genet 34:308-312

29. Geddis AE (2006) Inherited thrombocytopenia: congenital amegakaryocytic thrombocytopenia and thrombocytopenia with absent radii. Semin Hematol 43:196-203

30. Ihara K, Ishii E, Eguchi M et al (1999) Identification of mutations in the c-mpl gene in congenital amegakaryocytic thrombocytopenia. Proc Natl Acad Sci U S A 96:3132-3136

31. Guardiola P, Socie G, Pasquini R et al (1998) Allogeneic stem cell transplantation for Fanconi anaemia. Bone Marrow Transplant 21 (suppl 2):24-27
32. Guardiola Ph, Pasquini R, Dokal I et al (2000) Outcome of 69 allogeneic stem transplantations for Fanconi anemia using HLA-matched unrelated donors: a study on behalf of the European group for blood and marrow transplantation. Blood 95:422-429

33. de la Fuente J, Reiss S, McCloy M et al (2003) Non-TBI stem cell transplantation protocol for Fanconi anaemia using HLAcompatible sibling and unrelated donors. Bone Marrow Transplant 32:653-656

34. MacMillan ML, Wagner JE (2010) Haematopoietic cell transplantation for Fanconi anaemia-when and how. Br J Haematol 149 (1):14-21

35. de la Fuente J, Dokal I (2007) Dyskeratosis congenita: advances in the understanding of the telomerase defect and the role of stem cell transplantation. Pediatr Transplant 11:584-594

36. Dietz AD, Orchard PJ, Baker KS et al (2010) Disease-specific hematopoietic cell transplantation: nonmyeloablative conditioning regimen for dyskeratosis congenita. Bone Marrow Transplant 2010:1-7

37. Ahmed M, Dokal I (2009) Understanding aplastic anaemia/ bone marrow failure syndromes. Paediatr Child Health 19:351357 